Tropical Journal of Pharmaceutical Research October 2020; 19 (10): 2061-2066

ISSN: $1596-5996$ (print); 1596-9827 (electronic) (C) Pharmacotherapy Group, Faculty of Pharmacy, University of Benin, Benin City, 300001 Nigeria.

\title{
MiR-28-3p enhances healing of fracture via negative regulation of the target gene Sox6 and activation of PI3K/Akt signaling pathway
}

\author{
Wei Li, Xin Dong, Jian Zhao* \\ Department of Orthopedics, Tangdu Hospital, Air Force Military Medical University, Xi'an 710038, PR China \\ *For correspondence: Email: bpd85o@163.com \\ Sent for review: 18 June 2020 \\ Revised accepted: 28 September 2020
}

\begin{abstract}
Purpose: To investigate the effect of miR-28-3p on fracture healing, and the involvement of Sox6 gene and PI3K/Akt signaling pathway in the process.

Methods: Mouse osteoblast cell lines were cultured in vitro, and miR-28-3p over-expression and inhibitory plasmids were separately added to the medium. The corresponding control groups were set up. Real-time quantitative polymerase chain reaction (qRT-PCR) was used to measure the mRNA expressions of the osteogenesis-related genes Col1a1, Col- II and Col-X in osteoblasts. The protein expressions of Sox6, Col1a1, Col- II, Col-X, PI3K, p-PI3K, Akt and $p$-Akt in rat cartilage tissue were determined with Western blotting assay.

Results: The expression of Sox6 protein in the miR-28-3p over-expression group was significantly reduced, when compared with the miR-28 overexpression control, but Sox6 protein expression in the miR-28-3p inhibition group was significantly increased, relative to inhibition control group $(p<0.05)$. In the miR-28-3p over-expression and Sox6 over-expression groups, Col1a1 protein expression was significantly increased, while Col- II and Col-X protein expressions decreased, when compared with the respective over-expression control group $(p<0.05)$. Over-expression of miR-28-3p markedly upregulated phosphorylation levels of PI3K and Akt, relative to over-expression control group, while miR-28-3p inhibition significantly downregulated the phosphorylations of PI3K and Akt, relative to the inhibition control group $(p<0.05)$.

Conclusion: Over-expression of miR-28-3p may enhance the healing of fractures by induction of PI3K/Akt signaling route via negative regulation of the expression of Sox6 gene.
\end{abstract}

Keywords: MiR-28-3p, Sox6, PI3K/Akt signaling pathway, Fracture healing

\begin{abstract}
This is an Open Access article that uses a funding model which does not charge readers or their institutions for access and distributed under the terms of the Creative Commons Attribution License (http://creativecommons.org/licenses/by/4.0) and the Budapest Open Access Initiative (http://www.budapestopenaccessinitiative.org/read), which permit unrestricted use, distribution, and reproduction in any medium, provided the original work is properly credited.

Tropical Journal of Pharmaceutical Research is indexed by Science Citation Index (SciSearch), Scopus, International Pharmaceutical Abstract, Chemical Abstracts, Embase, Index Copernicus, EBSCO, African Index Medicus, JournalSeek, Journal Citation Reports/Science Edition, Directory of Open Access Journals (DOAJ), African Journal Online, Bioline International, Open-J-Gate and Pharmacy Abstracts
\end{abstract}

\section{INTRODUCTION}

Brittle fracture is a serious complication of osteoporosis, also known as osteoporotic fracture. Osteoporosis refers to fracture injury caused by a fall at, or below the center of gravity of the body. It results in relatively serious harm, usually in postmenopausal women, with adverse impact on the affected individuals and society. Due to dysfunction in osteoclasts and osteoblasts, bone resorption is greater than bone formation in patients with osteoporosis, resulting 
in rapid loss of bone mass and eventual reduction in bone strength [1]. In addition, due to decline in osteoblast cell function and decreased levels of growth factor, bone formation is insufficient during bone reconstruction, resulting in bone microdamage. The healing of fractures is a continuous process in which osteoblasts synthesize bone matrix, while osteoclasts absorb bone matrix and interact to ensure healing of the fracture [2]. Several investigations have confirmed that miRNAs are involved in regulatory roles in fracture healing during osteoblast differentiation and proliferation [3]. The miRNAs constitute a very important group of non-coding RNA which regulate the expressions of target genes in multicellular organisms by affecting the stability of mRNAs and their translation [4]. Studies have shown that miRNAs regulate balance between osteogenesis and osteoporosis, and are involved in the pathogenesis of osteoporosis [5]. In the present investigation, miR-28-3p was transfected into osteoblasts in order to study its effect on fracture healing, as well as the mechanisms involved.

\section{EXPERIMENTAL}

\section{Reagents and equipment}

Mouse osteoblasts were obtained from Shanghai Kanglang Biotechnology Co. Ltd. The reagents used, and their sources (in parenthesis) were: fetal bovine serum (Wuhan Punuosai Life Technology Co. Ltd); DMSO (Beijing Kairuiji Biotechnology Co. Ltd); RIPA tissue cell rapid lysis buffer (Shanghai Beinuo Biotechnology Co. Ltd.); BCA protein quantitative kit and developer powder (Shanghai Hengfei Biotechnology Co. Ltd); Bromophenol blue and ammonium dodecyl sulfonate (Shanghai Yuanye Biotechnology Co. Ltd); $30 \%$ acrylamide (Shanghai Sangon Bioengineering Co. Ltd); ECL kit (Shanghai Qiaoyu Biotechnology Co. Ltd), and protein antibodies (Abcam Company, USA).

The instruments/equipment used and their suppliers were: constant temperature incubator (Beijing Qinyeyongwei Technology Co. Ltd); electric homogenizer (Wuxi Depu instrument manufacturing Co. Ltd); Benchtop refrigerated centrifuge (Beijing Taize Jiaye Technology Development Co. Ltd); thermostatic culture oscillator (Wuxi MicroSep Biotechnology Co. Ltd); ultra-low temperature refrigerator (China Haier Group); vertical electrophoresis tank (Shanghai Zhennuo Biotechnology Co. Ltd); fluorescence microscope (Guangzhou Mingmei Optoelectronic Technology Co. Ltd), and ultrasonic pulverizer (Shanghai Tiancheng Technology Co. Ltd).
This research was approved by the Animal Ethical Committee of Department of Orthopedics, Tangdu Hospital, Air Force Military Medical University, Xi'an 710038, PR China, according to Principles of Laboratory Animal Care, with the approval number of 201845802 [6].

\section{Cell culture, transfection and grouping}

Osteoblasts were cultured in DMEM containing $15 \%$ serum, incubated in a $37^{\circ} \mathrm{C}$ constant temperature incubator with $\mathrm{CO}_{2}$, and subjected to digestion and passage. When the cell spacing increased and the cells became round (as observed under the microscope), a culture medium containing $15 \%$ bovine serum was added to terminate the digestion. Then, $2 \mu \mathrm{L}$ Lipo 2000 was dissolved in $100 \mu \mathrm{L}$ serum-free optiMEM medium and left at room temperature for $5 \mathrm{~min}$. Thereafter, miR-28-3p over-expression and inhibitory plasmids were added to the medium (separately), and the corresponding control groups were set up. The various groups were incubated at room temperature for $20 \mathrm{~min}$. The cells were divided into 4 groups, based on different transfection plasmids, and $400 \mu \mathrm{L}$ mixture was added to each well and replaced with a complete medium, followed with further culturing for $48 \mathrm{~h}$.

\section{Real-time polymerase reaction (qRT-PCR)}

Quantitative real-time PCR assay was used to determine the mRNA expressions of osteogenic genes in osteoblasts. Culture medium was removed from cells in logarithmic growth phase, and total RNA of rat cartilage tissues in each group was extracted with Trizol method. Reverse transcription was carried out using dilutions of the RNA samples. Real-time quantitative PCR assay was used for quantitative analysis of mRNA. Reverse transcription and PCR were performed at $42{ }^{\circ} \mathrm{C}$ for $50 \mathrm{~min}$, and incubation was done at $95{ }^{\circ} \mathrm{C}$ for $5 \mathrm{~min}$. Reverse transcriptase was inactivated at $42{ }^{\circ} \mathrm{C}$ for $50 \mathrm{~min}$ and at $95{ }^{\circ} \mathrm{C}$ for $5 \mathrm{~min}$. After the reaction, PCR amplification was performed using the prepared cDNA. The internal reference gene was GAPDH. The Ct value of each target gene was obtained. The PCR conditions were: pre-denaturation at $95^{\circ} \mathrm{C}$ for $10 \mathrm{~min}, 95^{\circ} \mathrm{C}$ for $15 \mathrm{~s}, 60{ }^{\circ} \mathrm{C}$ for $45 \mathrm{~s}$, and extension at $72^{\circ} \mathrm{C}$ for 2 min in 40 cycles, and storage at $4^{\circ} \mathrm{C}$. The relative quantification of each gene was carried out using the $2^{-\triangle \triangle C T}$ formula.

\section{Western blotting}

Protein expressions in rat cartilage tissue were assayed with Western blotting. Osteoblasts of each group in logarithmic growth phase were 
subjected to lysis with RIPA buffer containing protease inhibitor and phosphatase inhibitor PMSF. The protein content of each lysate was determined using BCA protein quantitative analysis. Then, protein $(30 \mu \mathrm{g})$ and $4 \times$ sample loading buffer were mixed and centrifuged to obtain a supernatant which was separated using SDS-polyacrylamide gel electrophoresis and transferred to PVDF membrane. The membrane was sealed by incubation with $10 \mathrm{~mL}$ of $5 \%$ skimmed milk powder at room temperature for $2 \mathrm{~h}$ at $4{ }^{\circ} \mathrm{C}$ overnight so as to block non-specific binding of the blot. Thereafter, the PVDF membrane was incubated with primary antibodies for Sox6, Col1a1, Col- II , Col-X, PI3K, $\mathrm{p}-\mathrm{PI} \mathrm{K}$, Akt and p-Akt at $4{ }^{\circ} \mathrm{C}$ overnight, after which TBST was used to wash off non-specific binding of primary antibodies from the membrane. Then, incubation of the membrane with secondary antibody at room temperature was done for 1 to $2 \mathrm{~h}$. Non-specific binding of secondary antibody was washed off, and ECL chemiluminescence was used for color development.

\section{Statistical analysis}

Data analysis was done with SPSS version. 20.0. Quantitative data are presented as mean \pm SD. Two-group comparison of mean values was done with independent sample $t$-test, while ANOVA was employed for comparison of mean values among multiple groups. Statistical significance was assumed at $p<0.05$.

\section{RESULTS}

Effect of miR-28-3p transfection on expressions of osteogenesis-related genes in osteoblasts

Over-expression of miR-28-3p resulted in significant increases in Col1a1 expression level and decreases in expression levels of Col- $\amalg$ and Col-X, relative to miR-28p over-expression control ( $p<0.05$ ). Compared to the inhibition control group, miR-28-3p inhibition group had marked decreases in Col1a1 expression level, and increases in expression levels of Col- II and Col-X $(p<0.05$; Table 1).

\section{Effect of miR-28-3p transfection on Sox6 protein expression in osteoblasts}

As shown in Figure 1, relative to the overexpression control group, the protein expression of Sox6 in the miR-28-3p over-expression group was significantly reduced, while, relative to the inhibition control, the expression of Sox6 protein in the miR-28-3p inhibition group was markedly elevated $(p<0.05)$.

Table 1: Effect of miR-28-3p transfection on expressions of osteogenesis-related genes in osteoblasts (mean $\pm \mathrm{SD}, \mathrm{n}=8$ )

\begin{tabular}{lccc}
\hline Group & $\begin{array}{c}\text { Col1a1 } \\
\text { mRNA }\end{array}$ & $\begin{array}{c}\text { Col- I } \\
\text { mRNA }\end{array}$ & $\begin{array}{c}\text { Col-X } \\
\text { mRNA }\end{array}$ \\
\hline Over- & $1.02 \pm$ & $1.12 \pm$ & $1.06 \pm$ \\
expression & 0.03 & 0.09 & 0.10 \\
control & & & \\
miR-28-3p & $4.11 \pm$ & $0.54 \pm$ & $0.60 \pm$ \\
over- & 0.23 & 0.06 & 0.09 \\
expression & $0.98 \pm$ & $1.03 \pm$ & $0.98 \pm$ \\
Inhibition & 0.11 & 0.10 & 0.17 \\
control & $0.60 \pm$ & $4.23 \pm$ & $3.57 \pm$ \\
miR-28-3p & 0.07 & 0.56 & 0.48 \\
inhibition & 0.07
\end{tabular}

${ }^{*} P<0.05$, vs over-expression control group; ${ }^{\#} p<0.05$, vs inhibition control group

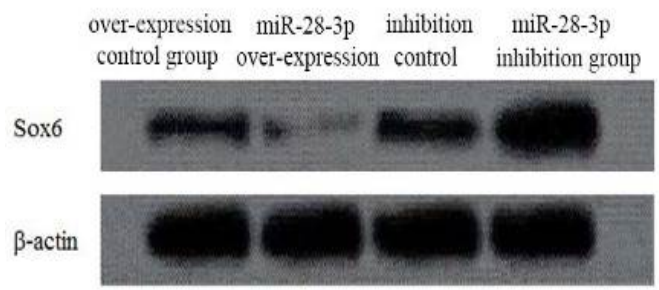

Figure 1: Effect of miR-28-3p transfection on Sox6 protein expression in osteoblasts

\section{Effect of Sox6 transfection on expressions of osteogenesis-related genes in osteoblasts}

The miR-28-3p over-expression group and Sox6 over-expression group had increased protein expressions of Col1a1 and markedly decreased protein expressions of Col- $\Pi$ and Col-X, when compared with the over-expression control group $(p<0.05)$. These results are presented in Figure 2 and Table 2.

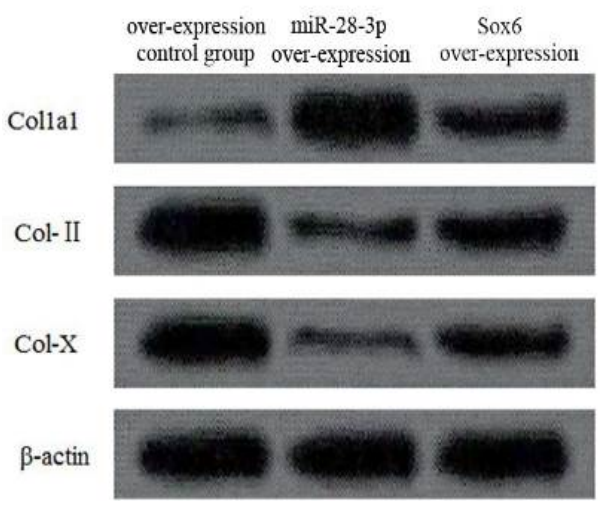

Figure 2: Effect of Sox6 transfection on expressions of osteogenesis-related genes in osteoblasts 
Table 2: Effect of Sox6 transfection on protein expression levels of Col1a1, Col- II and Col-X in osteoblasts (mean $\pm \mathrm{SD}, \mathrm{n}=8$ )

\begin{tabular}{lccc}
\hline Group & Col1a1 & Col-II & Col-X \\
\hline Over-expression & $1.02 \pm$ & $1.03 \pm$ & $1.15 \pm$ \\
control & 0.06 & 0.07 & 0.12 \\
miR-28-3p over- & $4.15 \pm$ & $0.41 \pm$ & $0.52 \pm$ \\
expression & $0.17^{*}$ & $0.12^{*}$ & $0.11^{*}$ \\
Sox6 over- & $3.44 \pm$ & $0.58 \pm$ & $0.63 \pm$ \\
expression & $0.21^{*}$ & $0.20^{*}$ & $0.17^{*}$ \\
\hline
\end{tabular}

${ }^{*} P<0.05$, vs over-expression control

\section{Effect of miR-28-3p transfection on PI3K/Akt signaling pathway}

Over-expression of miR-28p resulted in marked upregulation of phosphorylation levels of PI3K and Akt, when compared with the overexpression control, while, relative to the inhibition control, inhibition of miR-28-3p resulted in reduced phosphorylation levels of PI3K and Akt $(p<0.05$; Figure 3).

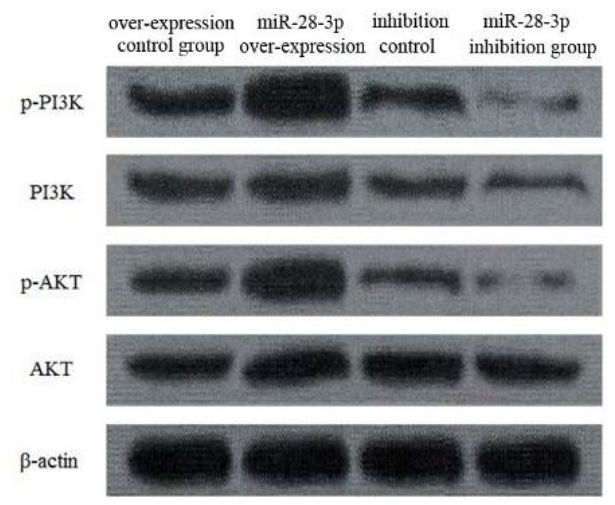

Figure 3: Effect of miR-28-3p transfection on PI3K/Akt signaling pathway

\section{DISCUSSION}

Osteoporotic fractures are the most serious consequences of osteoporosis, a disease which has a high prevalence in the elderly population. Osteoporosis reduces bone mass and bone mechanical strength, and results in loss of the normal weight-bearing capacity of the skeleton, leading to hip fractures and multiple vertebral fractures. Thus, osteoporosis is associated with high disability and mortality. It is considered a manifestation of bone failure [7].

Fractures are caused by bone fatigue and microfractures in the bone. Bone trabecula fracture and defects often impair the mechanical functions of bones. Bone trabecula forms the bone resorption lacunae which can result in punch and fracture, especially when the trabecular connection structure is missing. This results in disordered arrangement of internal collagen fibers and impairment of stress distribution and transmission which inevitably lead to reduction in the overall mechanical strength of bone, with attendant osteoporotic fractures [8]. The healing of osteoporotic fractures proceeds through the stages of repair and reconstruction during which osteoblastic absorption increases, while bone formation slows down. However, in postmenopausal women, estrogens significantly inhibit the proliferation and differentiation of osteoblasts, resulting in excessive loss of bone trabecula at the bone resorption site, lack of new bone formation, and decreased healing of fractures [9].

The miRNAs are non-coding RNAs with regulatory functions in eukaryotes. They have lengths in the range of 20 to 24 nucleotides, and exist in the non-coding region of eukaryote genomes. Each miRNA is complementary to the UTR region at the 3' end of target gene mRNA, and the regulation of gene expression is realized through inhibition of translation or mRNA cleavage [10]. Studies have shown that miRNAs have significant cell tissue specificity and may determine the behavioral changes in the growth and development of organisms [11]. In addition, studies have shown that miRNAs are significantly correlated with the etiologies of a variety of diseases (including cancer), and they act as oncogenes or as tumor suppressor genes, and participate in the regulation of cancer-related genomic regions or fragile sites [12].

Researchers have identified miRNAs with partial changes in expressions in osteoblasts, thereby confirming that miRNAs are associated with bone formation. In particular, miR-138 promotes osteoblastic differentiation. It has been reported that the over-expression of miR-138 reduced osteoblastic differentiation of mesenchymal stem cells in vitro, and reduced ectopic bone formation in vivo by $85 \%$, confirming that miR-138 plays a negative regulatory role in osteogenesis [13]. In addition, it has been found that the expression of miR-17-92 was down-regulated with differentiation of osteoblasts, indicating that miR17-92 is involved in the regulation of proliferation and differentiation of osteoblasts [13].

The miR-28-3p is a newly discovered miRNA which has been confirmed by studies to be involved in induction of cell proliferation and inhibition of cell apoptosis [14]. Col1a1 is expressed only in osteoblasts, and Col- II is the first protein encoded in chondrocyte differentiation and osteogenesis. The results of this study revealed that, relative to the over- 
expression control, miR-28-3p over-expression significantly increased Col1a1 expression level and decreased the expressions of Col- $I$ and Col-X, suggesting that miR-28-3p has potential effect on healing of fractures.

A previous study reported that Sox6, a member of the Sox transcription factor family, inhibited cell proliferation and apoptosis by up-regulating the expression of p53 [15]. Therefore, it was speculated, as part of the bases for this investigation, that the enhancement effect of miR-28-3p on fracture healing might be mediated through downregulation of Sox6. Interestingly, the results obtained indicated that, compared with the over-expression control group, the expression of Sox6 protein in the miR-28-3p over-expression group was significantly downregulated. Moreover, miR-28-3p overexpression and Sox6 over-expression resulted in significantly increased protein expression of Col1a1, and decreased protein expressions of Col- II and Col-X. These results suggest that miR-28-3p may indeed promote healing of fractures by targeted regulation of Sox6 gene expression. The PI3K/Akt signaling pathway, an ubiquitous pathway in cells, regulates cell proliferation and differentiation. It is enhanced by many known factors; for example, it is hyperactive in a variety of diseases, and it is involved in the reduction of apoptosis and promotion of cell proliferation [16]. The results of this study showed that miR-28-3p overexpression markedly upregulated the phosphorylation levels of PI3K and Akt, when compared with the over-expression control group. This confirms that the promotion influence of miR-28-3p on fracture healing is closely related to the PI3K/Akt signaling pathway.

\section{CONCLUSION}

The results obtained in this study demonstrate that over-expression of miR-28-3p may enhance healing of fractures through induction of PI3K/Akt signaling route via negative regulation of the expression of the target gene Sox6.

\section{DECLARATIONS}

\section{Conflict of interest}

No conflict of interest is associated with this work.

\section{Contribution of authors}

We declare that this work was done by the author(s) named in this article and all liabilities pertaining to claims relating to the content of this article will be borne by the authors. All authors read and approved the manuscript for publication. Jian Zhao conceived and designed the study. Wei Li, Xin Dong, Jian Zhao collected and analyzed the data, while Wei Li wrote the manuscript.

\section{Open Access}

This is an Open Access article that uses a funding model which does not charge readers or their institutions for access and distributed under the terms of the Creative Commons Attribution License (http://creativecommons.org/licenses/by/ 4.0) and the Budapest Open Access Initiative (http://www.budapestopenaccessinitiative.org/rea d), which permit unrestricted use, distribution, and reproduction in any medium, provided the original work is properly credited.

\section{REFERENCES}

1. Paul B, Terrence D, Clark WA. Vertebroplasty for acute painful osteoporotic fracture. Med J Aust 2017; 207(1): 279-281.

2. Dal-Ré $R$, Janiaud $P$, loannidis JPA. Real-world evidence: How pragmatic are randomized controlled trials labeled as pragmatic? BMC Med 2018; 16(1): 49.

3. Lopez YON, Garufi G, Seyhan AA. Altered levels of circulating cytokines and microRNAs in lean and obese individuals with prediabetes and type 2 diabetes. Mol Biosyst 2017; 13(1): 106-121.

4. Kurul NO, Ates F, Yilmaz I, Narli G, Yesildal C, Senkul T. The association of let-7c, miR-21, miR-145, miR-182, and miR-221 with clinicopathologic parameters of prostate cancer in patients diagnosed with low-risk disease. Prostate 2019; 79(10): 1125-1132.

5. Zhou Q, Shi C, Lv Y, Zhao C, Jiao Z, Wang T. Circulating microRNAs in Response to Exercise Training in Healthy Adults. Front Genet 2020; 11: 256.

6. World Health Organization. Principles of laboratory animal care. WHO Chron 1985; 39: 51-56.

7. Merlijn T, Swart KMA, van der Horst HE, Netelenbos JC, Elders PJM. Fracture prevention by screening for high fracture risk: a systematic review and meta-analysis. Osteoporos Int 2020; 31(2): 251-257.

8. Nakamae $T$, Fujimoto $Y$, Yamada $K$, Hiramatsu $T$, Hashimoto $T$, Olmarker $K$, Adachi N. Relationship between clinical symptoms of osteoporotic vertebral fracture with intravertebral cleft and radiographic findings. J Orthop Sci 2017; 22: 201-206.

9. Zhou J, Wang T, Zhao X, Miller DR, Zhai S. Comparative Efficacy of Bisphosphonates to Prevent Fracture in Men with Osteoporosis: A Systematic Review with Network Meta-Analyses. Rheumatol Ther 2016; 3(1): 117-128.

10. Weng SF, Hsu HR, Weng YL, Tien KJ, Kao HY. HealthRelated Quality of Life and Medical Resource Use in

Trop J Pharm Res, October 2020; 19(10): 2065 
Patients with Osteoporosis and Depression: A CrossSectional Analysis from the National Health and Nutrition Examination Survey. Int J Environ Res Public Health 2020; 17(3): 1124.

11. Coan RLB, Martins C. Landscape of Transposable Elements Focusing on the B Chromosome of the Cichlid Fish Astatotilapia latifasciata. Genes (Basel) 2018; 9(6): 269.

12. Proença MA, Biselli JM, Succi $M$, Severino FE, Berardinelli GN, Caetano A, Reis RM, Hughes DJ, Silva AE. Relationship between Fusobacterium nucleatum, inflammatory mediators and microRNAs in colorectal carcinogenesis. World J Gastroenterol 2018; 24(47): 5351-5365.

13. Elbakkoush AA, Khaleel A, Liu CT. MicroRNA and gene signature of severe cutaneous drug hypersensitivity reactions reveal the role of $\mathrm{miR}-483-5 p / m i R-28-5 p$ in inflammation by targeting Granulysin gene. Trop J Pharm Res 2017; 16(4): 771-779.

14. Huang $Y H$, Yang YL, Wang FS. The Role of miR-29a in the Regulation, Function, and Signaling of Liver Fibrosis. Int J Mol Sci 2018; 19(7): 1889.

15. De Pasquale V, Costanzo M, Siciliano RA, Mazzeo MF, Pistorio V, Bianchi L, Marchese E, Ruoppolo M, Pavone LM, Caterino M. Proteomic Analysis of Mucopolysaccharidosis IIIB Mouse Brain. Biomolecules 2020; 10(3): 355.

16. Spangle JM, Roberts TM, Zhao JJ. The emerging role of PISK/AKT-mediated epigenetic regulation in cancer. Biochim Biophys Acta Rev Cancer 2017; 1868(1): 123131. 